\title{
Medical History Start Date
}

National Cancer Institute

\section{Source}

National Cancer Institute. Medical History Start Date. NCI Thesaurus. Code C83128.

The date on which the medical history event begins. 